\title{
EXPERIMENTAL MALARIA IN MAN. III. THE CHANGES IN THE ELECTROCARDIOGRAM ${ }^{1}$
}

\author{
BY ERNST SIMONSON AND ANCEL KEYS
}

(From the Laboratory of Physiological Hygiene, University of Minnesota, Minneapolis)

(Received for publication July 22, 1949)

Electrocardiograms (ECGs) taken from patients with malaria are usually within normal limits or show only minor non-specific deviations. This is not evidence that changes do not occur or that the malarial process is without effect on the heart. The normal limits of the several ECG items are so wide, and are extending with the accumulation of more extensive data $(1,2)$, that definite and significant changes in an individual may occur without resulting in an ECG which by itself would be classified as abnormal. Obviously "before and after" studies are required.

The present report covers the ECG findings on 12 previously healthy young men before experimental blood-induced vivax malaria and on two occasions following the termination of the disease. Besides the usual resting ECG, the response of the ECG to the upright posture was studied. The subjects and conditions have been described in a previous paper (3).

\section{METHODS}

The three standard limb leads were recorded after a rest of at least ten minutes in the supine position on the tilt-table, the subject being clamped by the hips using Mayerson's (4) arrangement. The table was then tilted to an angle of $68^{\circ}$ and the ECG recordings were repeated during the first, fifth and tenth minute with the subject passively supported in this relatively upright position. All tests were made in the morning before breakfast. Control studies made several months before malaria were compared with repetitions on the second day and again three weeks after termination of the malaria.

The following ECG items were measured and analysed: Durations of P, QRS, P-R, and Q-T; the "constant" $K_{Q T}$ (QT divided by the square root of $R-R$ ); the amplitudes in each lead of $P, Q R S$, and $T$; the sums for the three leads of the amplitudes of QRS and of $T$

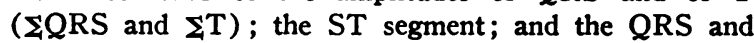
$T$ axes. From the simultaneous record of the heart

1 The results reported here were obtained in part under a contract, recommended by the Committee on Medical Research, between the Regents of the University of Minnesota and the Office of Scientific Research and Development. sounds, the duration of mechanical systole was estimated (interval between first and second sounds), and $\mathrm{K}_{\mathrm{gys}}$ was calculated as the quotient of this divided by. the square root of the $R-R$ interval. Since neither the $Q R S$ interval nor the amplitude or duration of $\mathbf{P}$ showed significant responses to malaria, they are not considered in the presentation of results below.

\section{RESULTS}

\section{In supine rest}

The findings in standard (basal) rest are summarized in Table I. The majority of the electrocardiographic items showed some change when they were recorded on the second day following the termination of malaria and these changes were statistically significant at the 5 per cent level for the P-R interval (slightly lengthened), for the sum of the QRS potentials in the three leads (increased), for $T_{2}$ (reduced but still positive), and for the angle of the QRS axis (small left axis shift). There was a more significant decline in the sum of the potentials of the $T$ waves in the three leads ( $\Sigma T$ ) ; the data indicate such a change could be expected by chance alone in less than one trial out of 100 (i.e., p. $=0.01$ ).

Three weeks after the termination of malaria some of the items had regressed toward the control but there were still some significant differences. The slight elevation of the heart rate was actually more significant at 21 days than at two days. It is of interest that the potentials of the $T$ waves, which had been depressed at two days, were elevated above the control at 21 days; there was "overshooting" in the regression toward the control in this item.

\section{Posture tests}

In the normal state, the passive change of the body to the upright posture results in certain electrocardiographic alterations which must be considered "normal" for this condition. For the present subjects these normal responses are sum- 
TABLE I

Means (12 men) for electrocardiographic items at rest before malaria, and after two and 21 days following

the termination of malaria

Differences between post-malaria and control values are given in the columns headed " $\Delta . "$ The statistical significances of the differences are indicated: ${ }^{*}=$ significant at the 5 per cent level, $\dagger=$ significant at the 1 per cent level.

\begin{tabular}{|c|c|c|c|c|c|}
\hline \multirow{3}{*}{ Item } & \multirow{3}{*}{$\begin{array}{c}\text { Control } \\
\text { Values }\end{array}$} & \multicolumn{4}{|c|}{ After malaria } \\
\hline & & \multicolumn{2}{|c|}{ Two days } & \multicolumn{2}{|c|}{21 days } \\
\hline & & Values & $\Delta$ & Values & $\Delta$ \\
\hline $\begin{array}{l}\text { Heart rate, beats } \\
\quad \text { per min. } \\
\mathrm{P}-\mathrm{R}, 0.01 \text { sec. } \\
\mathrm{K}_{\mathrm{QT}} \\
\mathrm{K}_{\mathrm{BYst}} \\
\Sigma \mathrm{RS}, \mathrm{mm} . \\
\mathrm{ST}_{2}, \mathrm{~mm} . \\
\mathrm{ST}_{3}, \mathrm{~mm} . \\
\mathrm{T}_{2}, \mathrm{~mm} . \\
\mathrm{T}_{3}, \mathrm{~mm} . \\
\Sigma \mathrm{T}, \mathrm{mm} . \\
\mathrm{QRSAxis, \text {degrees }} \\
\text { T Axis, degrees }\end{array}$ & $\begin{array}{c}51.6 \\
15.2 \\
0.376 \\
0.322 \\
27.3 \\
0.26 \\
-0.06 \\
3.51 \\
1.16 \\
7.74 \\
55.4 \\
45.2\end{array}$ & \begin{tabular}{|c|}
56.2 \\
16.1 \\
0.390 \\
0.320 \\
29.4 \\
0.10 \\
0 \\
2.80 \\
0.84 \\
5.77 \\
51.0 \\
44.0
\end{tabular} & $\begin{array}{c}4.6 \\
0.9^{*} \\
0.014 \\
-0.002 \\
2.1^{*} \\
0.16 \\
0.06 \\
-0.71^{*} \\
-0.32 \\
-1.97 \dagger \\
-4.4^{*} \\
-1.2\end{array}$ & \begin{tabular}{|l}
57.8 \\
15.9 \\
0.378 \\
0.330 \\
27.2 \\
0.07 \\
0 \\
4.27 \\
1.22 \\
8.72 \\
52.6 \\
45.8
\end{tabular} & $\begin{array}{c}6.2 \dagger \\
0.7 \\
0.002 \\
0.008 \\
-0.1 \\
-0.19 \\
0.06 \\
0.76 \dagger \\
0.06 \\
0.98^{*} \\
-2.8^{*} \\
0.6\end{array}$ \\
\hline
\end{tabular}

marized in the "Control" column in Table II as the differences between the values before and after ten minutes in the upright posture. From this material it is indicated that significant "normal" changes include increases in the heart rate and $\Sigma Q R S$; decreases in $K_{\text {syst }}, T_{2}, T_{3}$, and $\Sigma T$; and changes in the QRS axis (to the right) and in the $T$ axis (to the left).
TABLE II

Electrocardiographic response to the upright posture

Mean changes, upright minus supine, after ten minutes in the upright posture before and at two and 21 days following malaria.

Statistical significances are indicated as in Table $\mathbf{I}$.

In the "Control" column the significance indicated is that for the difference between supine and upright; in the other columns the significance relates to the difference between the posture effect in the control period and that in the post-malaria period.

\begin{tabular}{|c|c|c|c|}
\hline \multirow{2}{*}{ Item } & \multirow{2}{*}{ Control } & \multicolumn{2}{|c|}{ After malaria } \\
\hline & & Two days & 21 days \\
\hline $\begin{array}{l}\text { Heart rate, beats per min. } \\
\mathrm{P}-\mathrm{R}, 0.01 \text { sec. } \\
\mathrm{K}_{\mathrm{QT}} \\
\mathrm{K}_{\mathrm{syst}} \\
\mathrm{QQRS}_{\mathrm{Q}} \mathrm{mm} . \\
\mathrm{ST}_{2}, \mathrm{~mm} . \\
\mathrm{ST}_{3}, \mathrm{~mm} . \\
\mathrm{T}_{2}, \mathrm{~mm} . \\
\mathrm{T}_{3}, \mathrm{~mm} . \\
\mathrm{2T}, \mathrm{mm} . \\
\mathrm{QRS} \text { Axis, degrees } \\
\text { T Axis, degrees }\end{array}$ & $\begin{array}{c}14.7 \dagger \\
-0.75 \\
0.003 \\
-0.009^{*} \\
1.86^{*} \\
0.11 \\
0.03 \\
-1.02 \dagger \\
-0.82 \dagger \\
-1.90 \dagger \\
9.5 \dagger \\
-13.8^{*}\end{array}$ & $\begin{array}{c}39.5 \dagger \\
-2.08 \\
0.011 \\
0.010 \dagger \\
2.91 \\
-0.54^{*} \\
-0.62^{*} \\
-2.50 \dagger \\
-1.74 \dagger \\
-2.53 \\
8.9 \\
-67.4 \dagger\end{array}$ & $\begin{array}{c}23.2 \\
-2.10^{*} \\
0.014 \\
0.001 \\
1.52 \\
-0.01 \\
-0.12 \\
-2.07 \\
-1.18 \\
-3.48 \\
8.8 \\
-19.0\end{array}$ \\
\hline
\end{tabular}

Two days after the termination of malaria the assumption of the upright posture for ten minutes produced changes that differed significantly from those in the control period in several regards. The increase in the heart rate was greater, $\mathrm{K}_{\mathrm{syst}}$ increased rather than decreased, both $\mathrm{ST}_{2}$ and $\mathrm{ST}_{3}$ were depressed, the depression of $\mathrm{T}_{2}, \mathrm{~T}_{3}$ and $\Sigma \mathrm{T}$ was more marked, and the deviation of

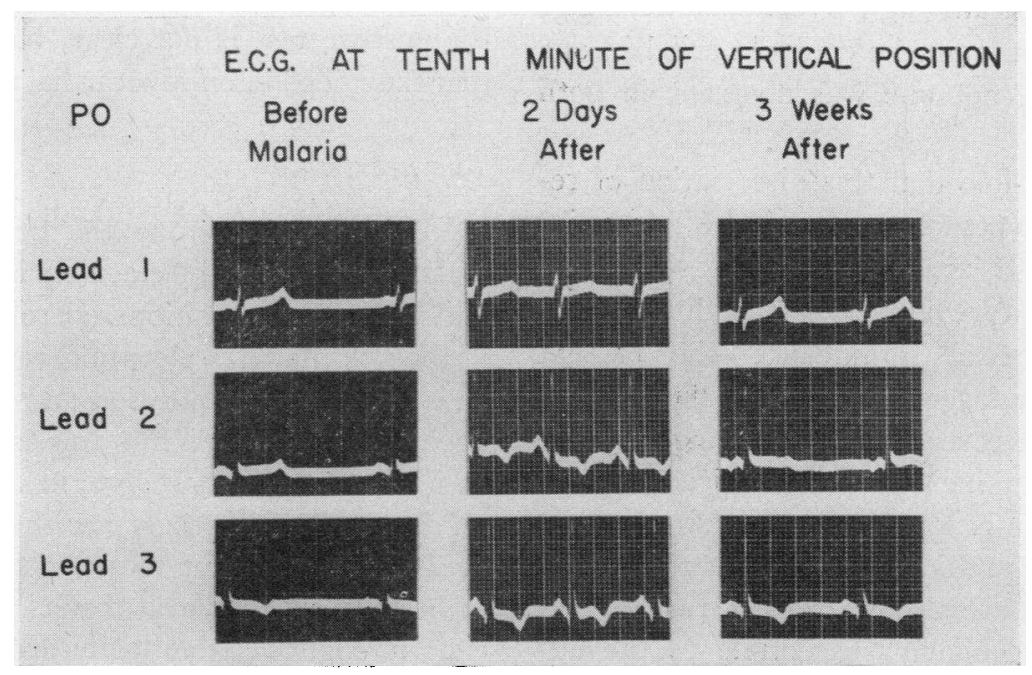

Fig. 1. Effect of Malaria on the Electrocardiogram after Ten Minutes in the Passive Upright $\left(68^{\circ}\right)$ Position 
the $\mathrm{T}$ axis to the left was greater. Three weeks after malaria the ECG response to the posture test was largely back to the control level, that is, the results did not differ significantly from the pre-malaria trial except for the single item of the $\mathrm{P}-\mathrm{R}$ interval in which the shortening effect of the upright posture was more marked (significant at the 5 per cent level) than in the control period. An example of the differences produced by malaria in the ECG at the tenth minute in the upright posture is given in Figure 1.

For all periods the ECG records were analysed in terms of the maximum change in the upright posture as well as for the change recorded after ten minutes. The results did not differ significantly from those summarized in Table II so that it appeared unwarranted to devote space here to the numerical data.

\section{GENERAL DISCUSSION}

It will have been observed that, with the exception of the $\mathrm{P}$ amplitude, $\mathrm{QRS}$ interval, and $\mathrm{K}_{\mathrm{QT}}$, every ECG item studied showed significant changes after malaria in either the basal (supine) records or in the upright posture. On the second day after the termination of malaria a significant decrease of $T_{2}$ appeared in both supine and posture tests but the significant "abnormalities" (deviations from control) in the other items appeared in only one of the two types of test. Nineteen days later the dissociation of the two test methods was complete; although five items were significantly different from the control period in one or the other test, not one was aberrant in both tests.

While it is possible that this dissociation of results in the two types of test would be less striking in studies with larger numbers of subjectswith the attendant reduction in the difficulty of establishing statistical significance-the present findings point to a large measure of independence in the phenomena revealed by the changes in the ordinary ECG (supine) and those elicited in the posture test. This is somewhat surprising at first sight. The first prediction might be that those ECG items which are altered in basal rest by malaria would be peculiarly susceptible to the additional stress of the upright posture and therefore the ECG in the upright position would be, in all respects, more aberrant than the ordinary ECG. Actually, the analysis of the posture test here, being made in terms of the ECG change from the supine to the upright position, does not make for a simple addition of the effects of malaria and the upright posture; only the added influence of malaria appears in the analysis.

Three weeks after the termination of malaria the ordinary (supine) ECG was still different from the control in four items but only one item, the $\mathrm{P}-\mathrm{R}$ interval, was aberrant in the posture test. This suggests that the special adjustment to posture was recovered more rapidly than the basic characteristics in simple horizontal rest. It is evident that the addition of the posture test in this study increased the information gained about the effect of malaria. This conforms to our general experience that tests involving some standardized stress always reveal more than tests limited to the ordinary resting state.

The response of the ECG to the vertical posture has been studied in some detail by Mayerson and Davis (4). Part of the change, of course, simply reflects the altered position of the heart in the chest. However, the tendency to right axis shift continues to progress during passive standing although the change in the position of the heart in the chest should be completed in a minute or less. Furthermore, progressive changes appear in other items, notably the depression of $\mathrm{T}$ and the increased angle between the QRS and the $\mathrm{T}$ axes. The full meaning of the changes in the ECG in the posture test is not clear, but it is improbable that they depend on myocardial anoxia $(4,5)$.

\section{The heart rate}

In the present study the heart rate following malaria was slightly elevated in both the supine and the upright positions. From Tables I and II it appears that this elevation was significant at 21 days in the supine position and at two days in the upright position. While this is true, it is not to be inferred that there were, in either position, significant differences between the rates at two and at 21 days. The proper conclusion would seem to be only that malaria results in a general tendency to slight relative tachycardia and that this is discernible for at least three weeks after termination of the infection and fever. 


\section{The duration of systole}

If we agree that, normally, the duration of systole is inversely proportional to the square root of the cycle length, then the present study indicates that the duration of electrical systole $(Q-T)$ was not affected significantly by malaria. The same is true for mechanical systole in the supine position but the situation is different in the posture test. The change to the upright posture normally produces a relative shortening of the time for mechanical systole. Two days after malaria, however, there was a relative lengthening of mechanical systole and the difference, as compared with the pre-malaria finding, was highly significant. On the face of it, this suggests a dissociation between electrical and mechanical systole but closer analysis does not support this idea. The mean relative duration of electrical systole was, in fact, prolonged in the posture test after malaria, but the prolongation is not quite enough to reach our criterion of significance. A reasonable conclusion would be that malaria tends to result in a slight relative prolongation of systole.

\section{The ST segment and the $T$ wave}

Tables I and II indicated a rather general tendency for malaria to result in depression of both the ST segment and the $T$ wave. The changes were usually small but in some cases involved a change in direction as well as simple reduction. For example, in the control period the upright posture produced a reduction in $T_{2}$ but this remained positive for all 12 men. On the second day after malaria, in the posture test $T_{2}$ became negative or small and diphasic (mainly inverted) in six of the men and this peculiarity persisted in a lesser degree after 21 days (see Figure 1).

\section{The electrical axes}

In supine rest malaria appeared to produce no change in the $T$ axis and only a slight change in the QRS axis. During postural adjustment, however, there was a very large left shift in the $T$ axis on the second day after malaria, but no significant change in the $Q R S$ axis. It is concluded that malaria produced a real change in the direction of repolarization in the upright posture, while the main direction of the impulse invasion was approximately unchanged.

\section{SUM MARY}

1. Electrocardiograms (three standard leads) were recorded from 12 men before blood-induced vivax malaria and on the second and 21st days following termination of the disease. On each occasion records were taken in basal rest (supine) and in the first, fifth and tenth minutes of passive elevation to $68^{\circ}$ on a hip-sling tilt-table.

2. Quantitative analysis showed that 12 out of 14 items measured were significantly changed by malaria, including a general tendency to depression of $\mathrm{T}$ and of the ST segment in leads II and III and, in the upright posture, a large shift of the $\mathrm{T}$ axis to the left.

3. The tilt-table was found useful in revealing changes not apparent from the electrocardiograms recorded in the ordinary supine posture.

4. Twenty-one days after malaria significant alterations from the pre-malaria control persisted in five electrocardiographic items.

5. Although most of the changes were statistically highly significant, they occurred within the so-called normal limits.

\section{BIBLIOGRAPHY}

1. Graybiel, A., McFarland, R. A., Gates, D. C., and Webster, F. A., Analysis of the electrocardiograms obtained from 1000 young healthy aviators. Am. Heart J., 1944, 27, 524.

2. Viscidi, P. C., and Geiger, A. J., Electrocardiographic observations on 500 unselected young adults at work. Am. Heart J., 1943, 26, 763.

3. Henschel, A., Taylor, H. L., and Keys, A., Experimental malaria in man. I. Physical deterioration and recovery. J. Clin. Invest., 1950, 29, 52.

4. Mayerson, H. S., and Davis, W. D., Jr., The influence of posture on the electrocardiogram. Am. Heart J., 1942, 24, 593.

5. Scherf, D., and Weissberg, J., The alterations of the $\mathrm{T}$-waves caused by a change of posture. Am. J. M. Sc., 1941, 201, 693. 